\title{
Report from the 3rd Cardiovascular Outcome Trial (CVOT) Summit of the Diabetes \& Cardiovascular Disease (D\&CVD) EASD Study Group
}

\author{
Oliver Schnell ${ }^{*}$, Eberhard Standl ${ }^{1}$, Doina Catrinoiu ${ }^{2}$, Stefano Genovese ${ }^{3}$, Nebojsa Lalic ${ }^{4}$, Katarina Lalic $^{4}$, \\ Jan Skrha ${ }^{5}$, Paul Valensi ${ }^{6}$ and Antonio Ceriello ${ }^{7,8}$
}

\begin{abstract}
The 3rd Cardiovascular Outcome Trial Summit of the Diabetes \& Cardiovascular Disease EASD Study Group was held on the 26-27 October 2017 in Munich. As in 2015 and 2016, this summit was organised in light of recently completed and published CVOTs on diabetes, aiming to serve as a reference meeting for in-depth discussions on the topic. Amongst others, the CVOTs EXSCEL, DEVOTE, the CANVAS program and the ACE-trial, which released primary outcome results in 2017, were discussed. Trial implications for diabetes management and recent perspectives of diabetologists, cardiologists, endocrinologists, nephrologists and general practitioners were highlighted. The clinical relevance of cardiovascular outcome trials and its implications regarding reimbursement were compared with real-world studies. The 4th Cardiovascular Outcome Trial Summit will be held in Munich 25-26 October 2018 (http://www.dcvd.org).
\end{abstract}

Keywords: CVOT Summit, D\&CVD EASD Study Group, Diabetes, Cardiovascular risk, CVOT, DEVOTE, CANVAS program, EXSCEL, ACE

\section{Background}

Patients with diabetes have an up to $50 \%$ increased risk of cardiovascular (CV) disease (CVD) compared to individuals without diabetes [1]. Optimisation of glycaemic control can reduce the long-term development of CVD and mortality $[2,3]$.

Driven by the Food and Drug Administration (FDA) and the European Medicines Agency (EMA), guidelines for the approval of novel glucose-lowering medications were published in 2008 and 2012, respectively $[4,5]$. These guidelines highlight, that new therapeutic approaches should not result in an increased CV risk. To rule out CV harm of novel treatment approaches, $\mathrm{CV}$ safety and benefits of glucose-lowering medications have therefore been the focus of cardiovascular outcome

\footnotetext{
*Correspondence: oliver.schnell@|rz.uni-muenchen.de

${ }^{1}$ Forschergruppe Diabetes e.V., Munich, Ingolstaedter Landstrasse 1, Neuherberg, 85764 Munich, Germany

Full list of author information is available at the end of the article
}

trials (CVOTs) in diabetes, which have been performed according to the suggestions of the abovementioned guidelines. In the CVOTs, combined primary $\mathrm{CV}$ endpoints are evaluated, which include CV mortality, nonfatal myocardial infarction (MI) and non-fatal stroke (3-point-MACE). Additional components could be e.g. hospitalisation for heart failure (HF), acute coronary syndrome and revascularisation procedures. Several CVOTs, which analysed DPP-4 inhibitors (saxagliptin, alogliptin, sitagliptin), GLP-1 receptor agonists (RA; lixisenatide, liraglutide, semaglutide) and SGLT-2 inhibitors (empagliflozin), were published until 2016 [6-12].

In 2017, CVOTs for canagliflozin (SGLT-2 inhibitor, CANVAS program), exenatide once weekly (GLP-1 RA, EXSCEL) and Insulin degludec (basal insulin analogue, DEVOTE) were published [13-16]. Also the ACE trial (acarbose), which focused on secondary prevention of CVD, was completed [17]. Other CVOTs, which included significant numbers of diabetic patients, investigated 
lipid-lowering strategies with evolocumab (FOURIER) and anacetrapib (REVEAL) $[18,19]$. As in 2015 and 2016 $[20,21]$, we present and summarise key aspects, which were discussed at the $3^{\text {rd }}$ CVOT Summit.

\section{Updates on CVOTs}

A summary of characteristics and results of CVOTs from 2017 is presented in Tables 1 and 2.

\section{SGLT-2 inhibitors}

The CANVAS program was a combination of two trials involving 10,142 participants with type 2 diabetes mellitus (T2DM) at high CV risk. It was shown that patients treated with the SGLT-2 inhibitor canagliflozin had a lower risk of $\mathrm{CV}$ events and a significant reduction of hospitalisation for HF. Although on the basis of the prespecified hypothesis testing sequence the renal outcomes are not viewed as statistically significant, the results showed a possible benefit of canagliflozin with respect to the progression of albuminuria and the composite outcome of a sustained $40 \%$ reduction in the estimated glomerular filtration rate (eGFR), the need for renalreplacement therapy or death from renal cause. The risk for amputation was increased compared to the control group [13].

\section{GLP-1 receptor agonists}

The results from the EXSCEL trial demonstrated CV safety in high risk subjects with T2DM who were treated with long-acting exenatide once weekly. With respect to safety the GLP-1 RA was non-inferior to placebo but not superior with respect to efficacy. The secondary outcomes were consistent with the primary outcome: CVD, fatal or nonfatal MI, fatal or nonfatal stroke as well as hospitalisation for HF and for acute coronary syndrome did not differ significantly between both groups [14]. There was, however, a $14 \%$ reduction in all-cause mortality with exenatide once weekly versus placebo (hazard ratio 0.86 , 95\% CI 0.77-0.97), although this difference could not be rated as significant due to the protocoldefined hierarchical order of statistical testing.

\section{Basal insulin}

Results of the DEVOTE trial indicate that for T2DM treatment, the ultra-long-acting, once-daily basal Insulin degludec is as safe in CV terms as Insulin glargine and associated with much lower rates of severe hypoglycaemia. Treatment with Insulin degludec resulted in a $27 \%$ reduction in the number of patients experiencing a severe episode, resulting in a $40 \%$ overall reduction of total episodes. Patients treated with Insulin degludec also experienced a 53\% reduction in the number of nocturnal episodes of severe hypoglycaemia [15].

\section{Alpha-glucosidase inhibitors}

In the ACE trial, pre-existing diabetes was an exclusion criterion and for inclusion participants required a history of CVD and impaired glucose tolerance. Designed as a CVOT with a 3-point-MACE, the ACE study could not achieve enough events, yielding a shift to an expanded 5-point-MACE [adding hospitalisation for HF and unstable angina (UA)] as primary outcome. The rate of events of the primary endpoint was not reduced, comparing acarbose with placebo. Also, most secondary outcomes did not show a difference between the two treatment groups. Confirming the primary result of the

Table 1 Overview of basic characteristics of CVOTs completed 2016/17 and published in 2017

\begin{tabular}{|c|c|c|c|c|c|c|c|c|c|}
\hline & $\begin{array}{l}\text { Study } \\
\text { status }\end{array}$ & Drug & Drug class & Intervention & Primary outcome & $\mathbf{N}$ & $\begin{array}{l}\text { Follow-up } \\
\text { (years) }\end{array}$ & $\begin{array}{l}\text { Start } \\
\text { and end } \\
\text { date }\end{array}$ & $\begin{array}{l}\text { Clinicaltrials. } \\
\text { gov ID }\end{array}$ \\
\hline $\begin{array}{l}\text { CANVAS } \\
\text { program }\end{array}$ & Completed & Canagliflozin & $\begin{array}{l}\text { SGLT-2 Inhibi- } \\
\text { tor }\end{array}$ & $\begin{array}{l}\text { Canagliflozin } \\
100 \text { mg vs. cana- } \\
\text { gliflozin } 300 \text { mg } \\
\text { vs. placebo }\end{array}$ & $\begin{array}{l}\text { CV death, MI, or } \\
\text { stroke }\end{array}$ & 10,142 & 3.6 & $\begin{array}{r}12.2009- \\
02.2017\end{array}$ & NCT01032629 \\
\hline EXSCEL & Completed & Exenatide & $\begin{array}{l}\text { GLP-1 recep- } \\
\text { tor agonist }\end{array}$ & $\begin{array}{l}\text { Exenatide once- } \\
\text { weekly vs. } \\
\text { placebo }\end{array}$ & $\begin{array}{l}\text { CV death, MI, or } \\
\text { stroke }\end{array}$ & 14,752 & 3.2 & $\begin{array}{r}06.2010- \\
04.2017\end{array}$ & NCT01144338 \\
\hline DEVOTE & Completed & $\begin{array}{l}\text { Insulin } \\
\text { degludec }\end{array}$ & Basal insulins & $\begin{array}{l}\text { Insulin deglu- } \\
\text { dec vs. Insulin } \\
\text { glargine }\end{array}$ & $\begin{array}{l}\text { CV death, Ml, or } \\
\text { stroke }\end{array}$ & 7637 & 2.0 & $\begin{array}{r}10.2013- \\
10.2016\end{array}$ & NCT01959529 \\
\hline ACE & Completed & Acarbose & $\begin{array}{l}\text { a-glucosidase } \\
\text { inhibitor }\end{array}$ & $\begin{array}{c}\text { Acarbose vs. } \\
\text { placebo }\end{array}$ & $\begin{array}{l}\text { CV death, MI, or } \\
\text { stroke, HHF,HUA }\end{array}$ & 6522 & 5.0 & $\begin{array}{r}02.2009- \\
04.2017\end{array}$ & NCT00829660 \\
\hline FOURIER & Completed & Evolocumab & $\begin{array}{l}\text { PCSK9 inhibi- } \\
\text { tor }\end{array}$ & $\begin{array}{l}\text { Evolocumab vs. } \\
\text { placebo }\end{array}$ & $\begin{array}{l}\text { CV death, MI, } \\
\text { stroke, UA or } \\
\text { coronary revascu- } \\
\text { larisation }\end{array}$ & 27,564 & 2.2 & $\begin{array}{r}01.2013- \\
11.2016\end{array}$ & NCT01764633 \\
\hline
\end{tabular}




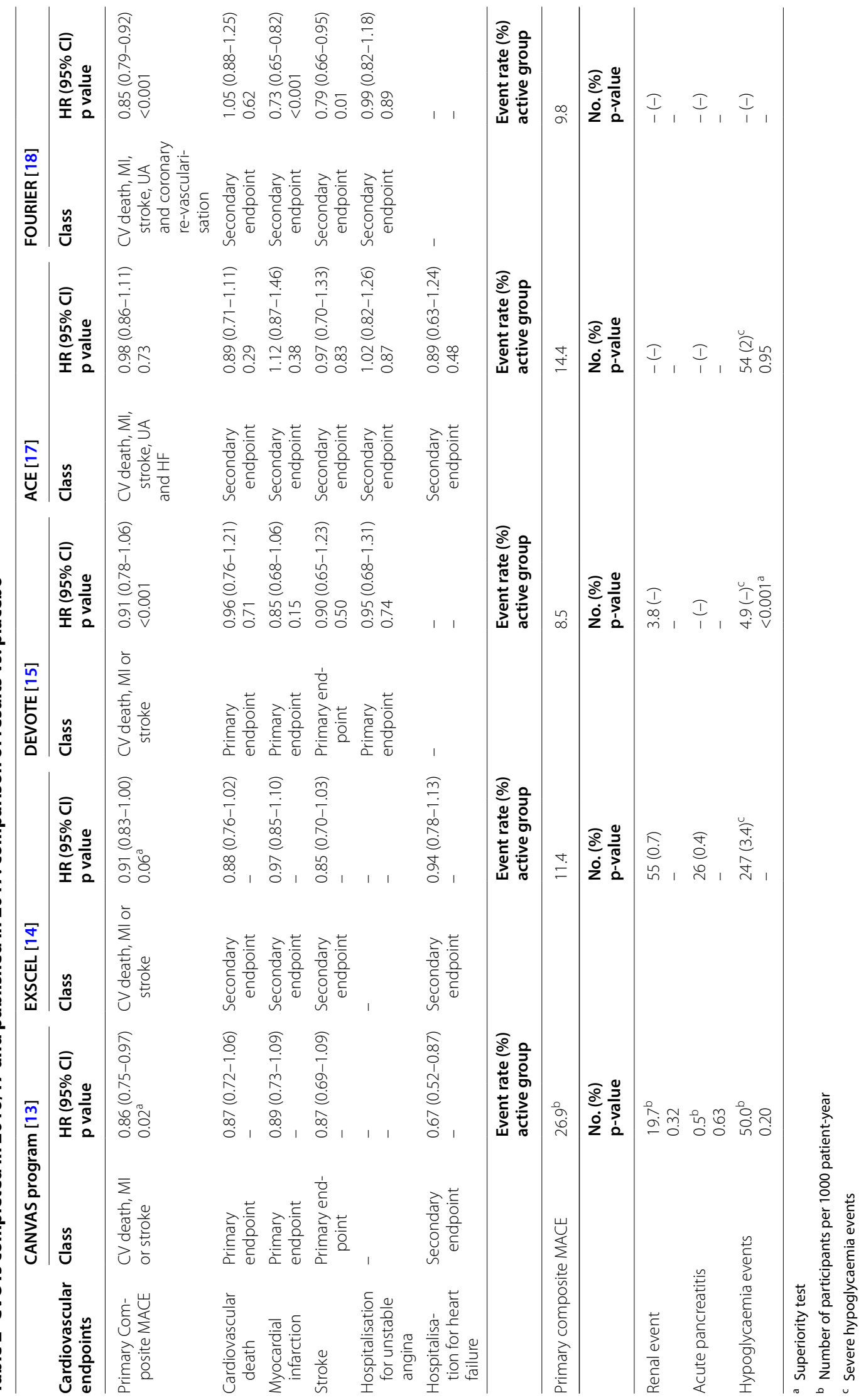


Stop-NIDDM trial [22], however, the number of participants who developed diabetes was reduced by $18 \%$ in the acarbose group compared to the placebo group [17].

\section{Lipid-lowering strategies}

The FOURIER trial demonstrated, that the PCSK9 inhibitor evolocumab on top of optimised statin therapy can reduce LDL-cholesterol to an unprecedented low level that was associated with a reduction of $\mathrm{CV}$ events. The positive outcomes on $\mathrm{CV}$ risk were especially apparent in patients with diabetes, though no effects on glucose level or HbA1c were observed. In contrast to statins, evolocumab did not increase the risk of new onset of diabetes, but this effect might be concealed by the relatively short follow-up time [18].

Even though the REVEAL trial provided positive results on another lipid-modifying medication-the CETP inhibitor anacetrapib-that reduced LDL-cholesterol and increased HDL-cholesterol levels, with a 9\% significant reduction in 3-point-MACE, the small increase in blood pressure and minor reduction in kidney function prevented the filing for approval by the FDA [19].

\section{Real-world data}

Apart from CVOTs the establishment of "real-world" data is an emerging field in diabetes. Regulatory bodies like FDA, the National Institute for Health and Care Excellence (NICE) or the German "Institut für Qualität und Wirtschaftlichkeit im Gesundheitswesen" (IQWiG) are demanding an increased inclusion and implementation of real world data to complement results of CVOTs. Studies based on electronic health records and registries could advance translational research and are often seen as a cost saving opportunity to improve patient care and population health.

Two examples concerning database-analysis were presented at the $3^{\text {rd }}$ CVOT Summit: CVD-REAL assessed data from more than 300,000 T2DM patients across six countries, $87 \%$ of whom did not have a history of CVD. These observational data showed that across this broad population treatment with SGLT-2 inhibitors (canagliflozin, dapagliflozin and empagliflozin) was significantly associated with a reduced overall rate of hospitalisation for HF by $39 \%$ and death from any cause by $51 \%$, compared to other treatment approaches. For the composite endpoint of hospitalisation for HF and death from any cause, a reduction of $46 \%$ was observed [23]. However, bias by drug indication cannot be totally excluded in CVD-REAL, as no clinical chemistry data were available for most patients and results could not be stratified for eGFR [23].

Data from the Swedish RiksSvikt Heart Failure registry from 2003 to 2011 was also presented. It included more than 36,000 patients with HF, of whom 8809 presented with diabetes. The database recorded about 70 variables and treatments managed by the Uppsala Clinical Research Centre. Data analysis revealed amongst others that diabetes compromises survival in HF irrespective of sex, HF aetiology or type. It increases mortality by $30-70 \%$ in diabetic patients. The prognosis is comparable between male and female diabetes patients and worst in those with systolic dysfunction and ischemic heart disease. It was concluded from the registry that special attention is required for diabetic patients with HF and strategies for improvement of outcome need to be established [24-26].

It was agreed that real-world evidences are beneficial to increase the understanding of the course of diabetes and its complications. A prerequisite for utilisation and interpretation of real-world data are high data quality and integrity of cases within the studied population. Nevertheless, discrepancies between randomised control trials and real-world data impede the comparison of such results. It was discussed that adherence to medication might differ between the two study designs and thereby contribute to contrasting results [27]. It was consensus that real-world data are valuable tools to complement, but not replace CVOTs. Nevertheless the role for real-world data will increase in the future.

\section{Key questions discussed during the 3rd CVOT Summit: How should results of CVOTs be reflected in guidelines?} The requirement for a balanced reflection of CVOT results in guidelines is undoubted. It was agreed that both national and international guidelines need to incorporate CVOT results in a swift and comprehensive manner, and that an adaption of international guidelines supports updates of guidelines on a local level [28]. Further strategies to distribute new CVOT results may also include online publications of guidelines and regular updates of medical standards.

In the future guidelines might need to distinguish between diabetic patients with and without CVD considering separate algorithms for treatment strategies of the two populations. Updated guidelines should not only reflect on medication that has demonstrated CV safety but also include examples of $\mathrm{CV}$ risk reduction. Updated guidelines should clearly discuss potential class effects of GLP-1 RAs, DPP-4 and SGLT-2 inhibitors, while at the same time highlight drug-specific effects observed in the outcome trials. Side effects and adverse events need to be included.

It was frequently suggested to provide joint guidelines by and for diabetologists, cardiologists and general practitioners (GPs). 
CVOTs in diabetes: are we looking at the right endpoints? Considering CVOTs from both a medical and regulatory perspective, the primary endpoint (MACE) was considered appropriate to demonstrate CV safety. This primary endpoint can be supported by more detailed secondary endpoints like time to first hospitalisation for HF or first occurrence of a microvascular event. Depending on the CVOT approach, additional, more specific endpoints may be considered. It has to be acknowledged that the robustness of some endpoints, e.g. the occurrence of an acute coronary syndrome, is still debated. Other endpoints like the occurrence and progression of diabetic retinopathy still need to be standardised to enable comparison between study outcomes. Overall, it was consensus that a clearer delineation of safety and efficacy components as well as better control of type 1 errors are required and that the implementation and publication of CVOTs should be standardised.

\section{Will diabetes with CVD in the future be treated by general practitioners, diabetologists, cardiologists or nephrologists?}

The general consensus was that comprehensive diabetes management is best to be performed by both diabetologists and GPs. Cooperation with cardiologists and nephrologists still needs to be optimised to deliver evidence-based medicine as a multi-disciplinary approach. Additionally, neurologists, psychiatrists, ophthalmologists and other specialities should be associated with the treatment of diabetes more closely. Thus, cross-functional medical education should be enhanced and guideline across associations should be aligned. Treatment decisions and recommendations should be shared more intensively between medical specialties and the primary care sector. In general, this was considered an important opportunity for establishing a network of cross-specialist collaborations in health care systems.

\section{Should we continue CV trials in the light of approved benefits?}

In general, the answer to this question was affirmative. Any medication for chronic disease should undergo longterm safety studies best carried out with standardised and clinically relevant endpoints. An additional CV or microvascular benefit was considered to be a pre-requisite for new approaches in the management of diabetes. Beside the high relevance of $\mathrm{CV}$ endpoints, microvascular endpoints should not be neglected. Studies focussing primarily on renal outcomes in diabetes are awaited in the near future.

Suggestions for future CVOTs were discussed. It was agreed that novel treatments should also be tested against treatment approaches, which have already shown to have proven $\mathrm{CV}$ benefit. It was acknowledged that it will be an increasing challenge to demonstrate an additional $\mathrm{CV}$ benefit. Suggestions included the need for mechanistic studies to further understand mechanisms responsible for $\mathrm{CV}$ and/or microvascular benefits as well as potential side effects. Studies should also help to clarify which group of diabetic patients benefits from specific treatment strategies or class of treatment and which diabetic patient groups do not.

\section{Conclusion}

The 3rd CVOT Summit of the D\&CVD EASD Study Group discussed key results of recent CVOTs and new studies on lipid-lowering strategies in an interactive multi-disciplinary format. The summit discussed both potentials and limitations of current CVOT designs as well as strategies for the implementation of CVOT results in treatment guidelines. It also discussed potential elements for future design of CVOTs in diabetes and shaped the role for real-world data. The D\&CVD EASD Study Group will continue its activity. In-depth discussions and presentations of upcoming CVOTs like CAROLINA, REWIND and DECLARE-TIMI, will be resumed at the 4th CVOT Summit, which will be held from 25 26 October 2018 in Munich (http://www.dcvd.org).

\begin{abstract}
Abbreviations
CV: cardiovascular; CVD: cardiovascular disease; CVOT: cardiovascular outcome trials; D\&CVD: Diabetes and Cardiovascular Disease; EASD: European Association of the Study of Diabetes; eGFR: estimated glomerular filtration rate; EMA: European Medicines Agency; FDA: Food and Drug Administration; GP: general practitioner; HF: heart failure; HHF: hospitalisation for heart failure; HUA: hospitalisation for unstable angina; IQWiG: Institut für Qualität und Wirtschaftlichkeit im Gesundheitswesen; MI: myocardial infarction; NICE: National Institute for Health and Care Excellence; RA: receptor agonist; T2DM: type 2 diabetes mellitus; UA: unstable angina.
\end{abstract}

\section{Authors' contributions}

OS, ES, DC, SG, NL, KL, JS, PV and AC contributed to the discussion and content of the report. All authors read and approved the final manuscript.

\footnotetext{
Author details

${ }^{1}$ Forschergruppe Diabetes e.V., Munich, Ingolstaedter Landstrasse 1, Neuherberg, 85764 Munich, Germany. ${ }^{2}$ Internal Medicine Department, Clinical County Emergency Hospital Constanta, Tomis Blvd. No. 145, 900591 Constanta, Romania. ${ }^{3}$ Diabetes, Endocrine and Metabolic Disease Unit, IRCCS Centro Cardiologico Monzino, Via Carlo Parea 4, 20138 Milan, Italy. ${ }^{4}$ Clinic for Endocrinology, Diabetes and Metabolic Diseases, Clinical Center of Serbia, Faculty of Medicine, University of Belgrade, Dr Subotica 13, Belgrade 11000, Serbia. ${ }^{5}$ 3rd Department of Internal Medicine, Charles University, 1st Faculty of Medicine, U Nemocnice 1, Praque 2128 08, Czech Republic. ${ }^{6}$ Department of Endocrinology Diabetology Nutrition, Paris 13 University, CINFO, CRNH-IdF, Jean VERDIER Hospital, Avenue du 14 Juillet, 93140 Bondy, France. ${ }^{7}$ Institut d'Investigacions Biomèdiques August Pi i Sunyer (IDIBAPS) and Centro de Investigación Biomedica en Red de Diabetes y Enfermedades Metabólicas Asociadas (CIBERDEM), Barcelona, Spain. ${ }^{8}$ Department of Cardiovascular and Metabolic Diseases, IRCCS MultiMedica, Via Milanese 300, 20099 Sesto San Giovanni, MI, Italy.
} 


\section{Acknowledgements}

We would like to thank all speakers and participants on the 3rd CVOT Summit for their active involvement in the scientific discussions leading to the present report. Moreover, we want to acknowledge the industry for their support of the meeting

\section{Competing interests}

The authors declare that they have no competing interests.

\section{Availability of data and materials}

Data sharing not applicable to this article as no datasets were generated during the current study.

\section{Consent for publication \\ Not applicable.}

\section{Ethics approval and consent to participate}

Not applicable.

\section{Funding}

No funding supported the generation of this manuscript.

\section{Publisher's Note}

Springer Nature remains neutral with regard to jurisdictional claims in published maps and institutional affiliations.

Received: 9 January 2018 Accepted: 22 January 2018

Published online: 19 February 2018

\section{References}

1. Dailey G. Overall mortality in diabetes mellitus: where do we stand today? Diabetes Technol Ther. 2011;13(Suppl 1):S65-74.

2. Holman RR, et al. 10-year follow-up of intensive glucose control in type 2 diabetes. N Engl J Med. 2008;359(15):1577-89.

3. Bergenstal RM. Glycemic variability and diabetes complications: does it matter? Simply put, there are better glycemic markers! Diabetes Care. 2015;38(8):1615-21.

4. FDA. Guidance for industry diabetes mellitus - evaluating cardiovascular risk in new antidiabetic therapies to treat type 2 diabetes. Washington, D. C.: US Department of Health and Human Services; 2008.

5. Guideline on clinical investigation of medicinal products in the treatment or prevention of diabetes mellitus. 2012. http://www.ema.europa. eu/docs/en_GB/document_library/Scientific_guideline/2012/06/ WC500129256.pdf. Accessed 26 Feb 2015.

6. White WB, et al. Alogliptin after acute coronary syndrome in patients with type 2 diabetes. N Engl J Med. 2013;369(14):1327-35.

7. Scirica BM, et al. Saxagliptin and cardiovascular outcomes in patients with type 2 diabetes mellitus. N Engl J Med. 2013;369(14):1317-26.

8. Green JB, et al. Effect of sitagliptin on cardiovascular outcomes in type 2 diabetes. N Engl J Med. 2015;373(3):232-42.
9. Pfeffer MA, et al. Lixisenatide in patients with type 2 diabetes and acute coronary syndrome. N Engl J Med. 2015;373(23):2247-57.

10. Marso SP, et al. Semaglutide and cardiovascular outcomes in patients with type 2 diabetes. N Engl J Med. 2016;375(19):1834-44.

11. Marso SP, et al. Liraglutide and cardiovascular outcomes in type 2 diabetes. N Engl J Med. 2016;375(4):311-22.

12. Zinman B, et al. Empagliflozin, cardiovascular outcomes, and mortality in type 2 diabetes. N Engl J Med. 2015;373(22):2117-28.

13. Neal B, et al. Canagliflozin and cardiovascular and renal events in type 2 diabetes. N Engl J Med. 2017;377(7):644-57.

14. Holman RR, et al. Effects of once-weekly exenatide on cardiovascular outcomes in type 2 diabetes. N Engl J Med. 2017;377(13):1228-39.

15. Marso SP, et al. Efficacy and safety of degludec versus glargine in type 2 diabetes. N Engl J Med. 2017;377(8):723-32.

16. Schnell $\mathrm{O}$, et al. Updates on cardiovascular outcome trials in diabetes. Cardiovasc Diabetol. 2017;16(1):128.

17. Holman RR, et al. Effects of acarbose on cardiovascular and diabetes outcomes in patients with coronary heart disease and impaired glucose tolerance (ACE): a randomised, double-blind, placebo-controlled trial. Lancet Diabetes Endocrinol. 2017:S2213-8587(17):30309.

18. Sabatine MS, et al. Evolocumab and Clinical Outcomes in Patients with Cardiovascular Disease. N Engl J Med. 2017:376(18):1713-22.

19. H.T.R.C. Group. Effects of anacetrapib in patients with atherosclerotic vascular disease. N Engl J Med. 2017;377(13):1217-27.

20. Schnell O, et al. Report from the 1st Cardiovascular Outcome Trial (CVOT) Summit of the Diabetes \& Cardiovascular Disease (D\&CVD) EASD Study Group. Cardiovasc Diabetol. 2016;15:33.

21. Schnell O, et al. Report from the 2nd Cardiovascular Outcome Trial (CVOT) Summit of the Diabetes and Cardiovascular Disease (D\&CVD) EASD Study Group. Cardiovasc Diabetol. 2017;16(1):35.

22. Chiasson $\mathrm{JL}$, et al. Acarbose for prevention of type 2 diabetes mellitus: the STOP-NIDDM randomised trial. Lancet. 2002;359(9323):2072-7.

23. Kosiborod $M$, et al. Lower risk of heart failure and death in patients initiated on SGLT-2 inhibitors versus other glucose-lowering drugs: the CVD-REAL Study. Circulation. 2017:136(3):249-59.

24. Johansson I, et al. Prognostic implications of type 2 diabetes mellitus in ischemic and nonischemic heart failure. J Am Coll Cardiol. 2016;68(13):1404-16.

25. Johansson I, et al. Risk factors, treatment and prognosis in men and women with heart failure with and without diabetes. Heart. 2015;101(14):1139-48.

26. Johansson I, et al. Is the prognosis in patients with diabetes and heart failure a matter of unsatisfactory management? An observational study from the Swedish Heart Failure Registry. Eur J Heart Fail. 2014;16(4):409-18.

27. Carls GS, et al. Understanding the gap between efficacy in randomized controlled trials and effectiveness in real-world use of GLP-1 RA and DPP-4 therapies in patients with type 2 diabetes. Diabetes Care. 2017:40(11):1469-78

28. Standl $E$, et al. Integration of recent evidence into management of patients with atherosclerotic cardiovascular disease and type 2 diabetes. Lancet Diabetes Endocrinol. 2017:5(5):391-402.

\section{Submit your next manuscript to BioMed Central and we will help you at every step:}

- We accept pre-submission inquiries

- Our selector tool helps you to find the most relevant journal

- We provide round the clock customer support

- Convenient online submission

- Thorough peer review

- Inclusion in PubMed and all major indexing services

- Maximum visibility for your research

Submit your manuscript at www.biomedcentral com/submit

Ciomed Central 